\title{
ENFERMAGEM

\section{Qual é a agenda de pesquisa de Enfermagem para a pandemia COVID-19?}

\author{
Erica Gomes Pereira ${ }^{1}$ \\ Adriana Maria da Silva Felix ${ }^{2}$ \\ Lúcia Yasuko Izumi Nichiata ${ }^{1}$ \\ Maria Clara Padoveze
}

${ }^{1}$ Universidade de São Paulo, Escola de Enfermagem, Departamento de Enfermagem em Saúde Coletiva, São Paulo, SP, Brasil. E-mail: egpereira@usp.br

${ }^{2}$ Santa Casa de São Paulo, Faculdade de Ciências Médicas, São Paulo, SP, Brasil.

Como citar este artigo:

Pereira EG, Felix AMS, Nichiata LYI, Padoveze MC. What is the Nursing research agenda for the COVID-19 pandemic? Rev Esc Enferm USP. 2020;54:e03661. doi: https://doi.org/10.1590/S1980-220X2020pv0103661

Em 2020 comemoramos 200 anos desde o nascimento de Florence Nightingale, fundadora da enfermagem profissional, pioneira no uso das estatísticas, e promotora de reformas sociais que precederam estados de bem-estar ${ }^{(1)}$.

Em 2018, para comemorar esse bicentenário, a Organização Mundial da Saúde (OMS) em colaboração com o Conselho Internacional de Enfermagem (CIE) lançou a campanha Nursing Now para impulsionar o debate sobre o perfil de enfermagem em todo o mundo ${ }^{(2)}$. A Assembleia Mundial de Saúde designou 2020 como o Ano Internacional dos Profissionais de Enfermagem e Obstetrícia, convocando os líderes mundiais a fazer investimentos maciços, a fim de melhorar e apoiar o desempenho desses profissionais que enfrentam os desafios de saúde do século $\mathrm{XXI}^{(3-4)}$. Sobretudo, neste exato ano, os profissionais de saúde, em particular, os enfermeiros, tornaram-se o centro das atenções devido ao advento da COVID-19, oficialmente declarada como pandemia pela OMS em 11 de março de $2020^{(5)}$.

O papel essencial dos profissionais de saúde durante essa pandemia é um marco na história contemporânea. Entre muitas contribuições da enfermagem no contexto da saúde, têm sido observadas atuações relevantes na educação em saúde, prevenção de infecções e vigilância de doenças, organização e preparação de serviços de longo prazo, proteção de pessoas com doenças crônicas, bem como cuidados agudos de casos graves com COVID-19(6-9).

Como a maior categoria de profissionais de saúde em todo o mundo ${ }^{(10)}$, os enfermeiros têm feito um trabalho monumental, cada vez mais baseado em evidências. No entanto, entre os numerosos pedidos de financiamento de pesquisa para apoiar a resposta à COVID-19, pelo nosso conhecimento, nenhum foi identificado para ser especificamente projetado para auxiliar na pesquisa de enfermagem. $\mathrm{Na}$ rápida efervescência de vários grupos de pesquisa que se acumularam, tudo parece indicar que a pesquisa de enfermagem permanece subsumida no conhecimento de outras disciplinas ${ }^{(10)}$. Em grupos multidisciplinares de pesquisadores em saúde pública, isso pode ser visto como um ganho. No entanto, também pode ser uma perda no que diz respeito ao conhecimento de enfermagem, e, além disso, em pontos de prestígio na arena de tomada de decisão e, principalmente, oportunidades de qualificação do cuidado de enfermagem prestado.

Paralelamente à participação em equipes multidisciplinares, os grupos de pesquisa de enfermagem devem construir parcerias entre si, a fim de fortalecer as capacidades de pesquisa que atendam à nossa especificidade, preferencialmente em grupos multinacionais, a fim de ampliar o potencial de generalização dos resultados e captação de recursos.

Nosso objetivo é convocar os enfermeiros a trabalhar em conjunto ao longo de uma agenda de prioridades de pesquisa na enfermagem com foco na pandemia COVID-19. Em rápida revisão das pesquisas realizadas por enfermeiros, identificamos temas e subtemas de interesse, e propusemos alguns que consideramos mais relevantes para serem desenvolvidos em pesquisa de enfermagem (Quadro 1). 
PONTO DE VISTA
Quadro 1 - Possíveis temas e subtemas para pesquisa em enfermagem sobre COVID-19.

1 - Aspectos psicossociais

- Ansiedade, estigma, estresse e enfrentamento das equipes de enfermagem na gestão da COVID-19;

- Experiências de enfermeiros com as equipes multidisciplinares no cuidado da COVID-19;

- Experiências de enfermeiros na implantação e monitoramento de programas de biossegurança para a reabertura de escolas, faculdades, entre outros;

- Comunidades vulneráveis e a experiência de enfermeiros com organizações comunitárias para a prevenção e gestão da COVID-19;

- O papel da mídia e das redes sociais como fontes de informação na disseminação de ansiedade e medo, e seu impacto nas equipes de enfermagem.

2 - Políticas e serviços de saúde

- Operacionalização dos protocolos clínicos de enfermagem para COVID-19;

- Cuidados de enfermagem durante a pandemia COVID-19 e a interação com o monitoramento e cuidado de outras doenças transmissíveis, doenças não transmissíveis, drogadição, saúde infantil e materna, violência urbana, saúde mental, saúde bucal, saúde sexual e reprodutiva;

- Adaptação de programas de biossegurança na assistência à enfermagem para reduzir a disseminação da COVID-19;

- Definição de prioridades nacionais para a pesquisa de enfermagem nos sistemas de saúde.

3 - Tecnologias em Saúde

- Telenfermagem durante a pandemia COVID-19 como forma de monitorar casos suspeitos e confirmados de COVID-19 e condições relacionadas a outras doenças transmissíveis, doenças não transmissíveis, drogadição, saúde infantil e materna, violência urbana, saúde mental, saúde bucal, saúde sexual e reprodutiva;

- Desenvolvimento de aplicativos para suporte clínico remoto no cuidado de enfermagem durante a pandemia COVID-19.

4 - Epidemiologia da COVID-19

- Perfis populacionais dos atingidos em áreas remotas ou populações vulneráveis;

- Prevalência, incidência e fatores de risco da COVID-19 entre profissionais de enfermagem;

- Custo-efetividade dos equipamentos de proteção individual entre profissionais de enfermagem;

- Experiência das equipes de enfermagem na limitação da transmissão e gerenciamento de casos de COVID-19;

- Barreiras e capacitadores para a adesão às medidas preventivas da COVID-19 entre os profissionais de enfermagem.

5 - Pesquisa e Educação em Enfermagem

- Metodologias de pesquisa de enfermagem aplicáveis na situação de confinamento devido à COVID-19;

- Estratégias inovadoras de ensino-aprendizagem e impacto na assistência à enfermagem durante a pandemia;

- Estratégias para identificar, monitorar e mitigar a ansiedade, o estresse e o medo dos estudantes de enfermagem devido à pandemia COVID-19;

- Atenção à educação em saúde dos pacientes e seus familiares para a prevenção e gestão do COVID-19 em diferentes contextos.

6 - Gerenciamento em Enfermagem

- Gerenciamento das competências da equipe de enfermagem durante emergências sanitárias como a pandemia COVID-19;

- Dilemas éticos da equipe de enfermagem na gestão de casos e cuidados post-mortem durante a pandemia COVID-19;

- Gestão de equipamentos de proteção individual em ambientes especiais como clínicas de hemodiálise, obstetrícia, neonatologia, entre outros.

Não pretendemos abranger todos os aspectos da pesquisa necessários para o combate à COVID-19, uma vez que isso tem sido amplamente discutido em cenários internacionais ${ }^{(10-11)}$. A intenção é provocar a reflexão e estimular o pensamento global sobre pesquisas de enfermagem relacionadas à COVID-19.

A agenda proposta convida enfermeiros de nível nacional e internacional a combinar esforços, engajar associações e sociedades, instituições de ensino de enfermagem e agências de fomento para promover a visibilidade da produção de conhecimentos de enfermagem. O objetivo final é abordar o direito universal para acesso aos cuidados de saúde. Assim, a pesquisa de enfermagem do século XXI fortalecerá o legado de Florence Nightingale.

\section{REFERÊNCIAS}

1. Thompson DR, Darbyshire P. Nightingale's year of nursing: rising to the challenges of the COVID-19 era. BMJ. 2020;370:m2721. doi: https://doi.org/10.1136/bmj.m2721

2. World Health Organization. Nursing now campaign [Internet]. Geneva: WHO; 2020 [cited 2020 Sep 16]. Available from: https://www.who.int/hrh/news/2018/nursing_now_campaign/en/

3. World Health Organization. Year of the nurse and the midwife [Internet]. Geneva: WHO; 2020 [cited 2020 Sep 20]. Available from: https://www.who.int/campaigns/year-of-the-nurse-and-the-midwife-2020

4. Crisp N, Iro E. Nursing Now campaign: raising the status of nurses. Lancet. 2018;391(10124):920-21. doi: https:// doi.org/10.1016/S0140-6736(18)30494-X

5. World Health Organization. Timeline of WHO's response to COVID-19 [Internet]. Geneva: WHO; 2020 [cited 2020 Sep 16]. Available from: https://www.who.int/news-room/detail/29-06-2020-covidtimeline

6. Chen SC, Lai YH, Tsay SL. Nursing perspectives on the impacts of COVID-19. J Nurs Res. 2020;28(3):e85. doi: https://doi.org/10.1097/NRJ.0000000000000389

7. Huang L, Lei W, Xu F, Liu H, Yu L. Emotional responses and coping strategies in nurses and nursing students during Covid-19 outbreak: a comparative study. PLoS One. 2020;15(8):e0237303. doi: https://doi.org/10.1371/ journal.pone.0237303 
PONTO DE VISTA
8. Courtenay M, Burnett E, Castro-Sanchez E, Figueiredo RM, Toit B, Gallagher R, et al. Preparing nurses for COVID-19 response efforts through involvement in antimicrobial stewardship programmes. J Hosp Infect. 2020;106(1):176-8. doi: https://doi.org/10.1016/j.jhin.2020.06.011

9. Osingada CP, Porta CM. Nursing and Sustainable Development Goals (SDGs) in a COVID 19 world: the state of the science and a call for nursing to lead. Public Health Nurs. 2020;37(5):799-805. doi: https://doi.org/10.1111/ phn. 12776

10. World Health Organization. COVID-19 Public Health Emergency of International Concern (PHEIC). Global research and innovation forum: towards a research roadmap [Internet]. Geneva: WHO; 2020 [cited 2020 Sep 15]. Available from: https://www.who.int/publications/m/item/covid-19-public-health-emergency-of-internationalconcern-(pheic)-global-research-and-innovation-forum

11. World Health Organization. A Coordinated Global Research Roadmap: 2019 Novel Coronvirus [Internet] Geneva: WHO; 2020 [cited 2020 Sep 16]. Available from: https://www.who.int/blueprint/priority-diseases/keyaction/Coronavirus_Roadmap_V9.pdf?ua=1 